\title{
Energy and System Size Dependence of Strangeness Production from SPS to RHIC
}

\author{
J. Takahashi for the STAR Collaboration \\ Instituto de Física Gleb Wataghin, Universidade Estadual de Campinas, Brazil \\ E-mail: jun@ifi.unicamp.br
}

\begin{abstract}
Strange particle production is an important experimental observable that allows the study of the strongly interacting matter created in relativistic heavy-ion collisions. The STAR experiment at RHIC has a unique capability of measuring identified strange particles over a wide range of acceptance providing a rich set of data to perform a systematic study. In addition to the data from $\mathrm{Au}+\mathrm{Au}$ collisions, strange particles from $\mathrm{p}+\mathrm{p}$ and $\mathrm{d}+\mathrm{Au}$ collisions are also available for comparison and normalization. A new set of data from $\mathrm{Cu}+\mathrm{Cu}$ reactions at $62 \mathrm{GeV}$ and 200 $\mathrm{GeV}$ provides the chance to compare the system size dependence observed in $\mathrm{Au}+\mathrm{Au}$ collisions with this smaller system size. In addition to the comparison of the yields, a statistical thermal model was used to extract freeze-out characteristics for the different system sizes and collision energies.
\end{abstract}

\section{Introduction}

Relativistic heavy-ion collisions provide the ideal environment with which to study strongly interacting nuclear matter at different thermodynamic conditions. Lattice QCD calculations predict that above a certain critical temperature, nuclear matter undergoes a phase transition forming a deconfined state of quarks and gluons [1]. Various signatures of QGP formation have been proposed with the enhancement of strangeness production being one of the experimental observables [2, 3]. Despite the large amount of different signatures that were proposed, due to the complexity of the different processes that might be competing in the reaction, a clear and unique signal of the QGP formation is not possible. Thus, to understand and characterize the system formed in these heavyion collisions, it is necessary to perform a systematic study of the production of various particles including energy dependence and system size dependence as well as comparison to other reactions such as $\mathrm{p}+\mathrm{p}, \mathrm{d}+\mathrm{Au}$ and $\mathrm{Cu}+\mathrm{Cu}$. This kind of complete study is only possible now due to the large amount of data accumulated from several years of RHICBNL operations and the new analysis from the lower energy CERN-SPS experiments.

STAR [4 has a complex system of detectors specialized in measuring the maximum number of particles produced in a RHIC collision on an event by event basis. In particular, the tracking detectors from STAR have a high efficiency for measuring charged particle tracks at mid-rapidity. The excellent track resolution and primary 
vertex position resolution yields a high efficiency for reconstructing strange particles through their secondary vertex and cascade decay topologies. In general, the invariant mass peaks of these particles can be extracted with good signal to noise ratios by applying only some basic geometrical and track quality cuts. Energy loss (dE/dx) information of the daughter tracks is also used to reduce combinatorial background. The background under the invariant mass peak can be subtracted by fitting the background shape or by simply counting the neighboring bins. Transverse momentum spectra for these strange particles are obtained by calculating the invariant mass for each $p_{T}$ bin and correcting for inefficiency and acceptance on a point by point basis. The efficiency and acceptance corrections are calculated using simulated particles embedded into real events. The total yield ( $\mathrm{dN} / \mathrm{dy})$ is obtained by using Maxwell-Boltzmann or exponential curves to extrapolate into the low and high $p_{T}$ region that is not covered by the measurement. Systematic errors are estimated considering several factors in the different steps of the analysis such as the variation in the yield due to variations in the background subtraction techniques, variations due to differences in the topological geometric cuts and differences between real data and simulated data in the efficiency calculation.

Here in this article, we present an analysis that concentrates on the production of strange particles, with special interest in the energy and system size dependence comparing the particle yields with predictions obtained from a statistical thermal model. Data presented here are a compilation of STAR experiment data from the last seven years [5, 6, 7] and some additional data points in the lower energy range from experiments NA49 [8] and NA57 [9] obtained from the literature.

\section{Size Dependence}

The large amount of data measured for each reaction system also allows for the classification of events based on the centrality of the collision. Collision centrality is determined by the measured charged particle multiplicities. For each centrality one can estimate the average number of particles that participate in the reaction $\left\langle N_{\text {part }}\right\rangle$ using a Glauber Model calculation, that also provides the equivalent number of binary collisions $\left\langle N_{b i n}\right\rangle$. Figure 1a shows the yields of $\Lambda, \bar{\Lambda}, \Xi^{ \pm}$and $\Omega^{ \pm}$as a function of $\left\langle N_{\text {part }}\right\rangle$ for all the studied centralities in $\mathrm{Au}+\mathrm{Au}$ and $\mathrm{Cu}+\mathrm{Cu}$ collisions at $\sqrt{s_{N N}}=200 \mathrm{GeV}$. A detailed discussion on $K_{S}^{0}$ and $\Lambda$ production in $\mathrm{Au}+\mathrm{Au}$ and $\mathrm{Cu}+\mathrm{Cu}$ collisions as a function of system size can be found elsewhere [7, 10]. Most of the $\mathrm{Cu}+\mathrm{Cu}$ data seem to be consistent with $\mathrm{Au}+\mathrm{Au}$ yields when compared using the system volume $\left(\left\langle N_{\text {part }}\right\rangle\right)$. The yields of $\Lambda$ hyperons for the most central $\mathrm{Cu}+\mathrm{Cu}$ data seem to be slightly higher than the equivalent $\mathrm{Au}+\mathrm{Au}$ data (around 30\%). A possible explanation for this behavior could be found with a different scaling. A more detailed discussion was presented elsewhere at this conference [10].

To evaluate the strangeness enhancement in heavy-ion collisions, $\mathrm{Au}+\mathrm{Au}$ data is

compared to $\mathrm{p}+\mathrm{p}$ collisions normalized by $\left\langle N_{\text {part }}\right\rangle$. Figure 1 $\mathrm{b}$ shows the normalized yields as a function of $\left\langle N_{\text {part }}\right\rangle$ for all centralities in $\mathrm{Au}+\mathrm{Au}$ and $\mathrm{Cu}+\mathrm{Cu}$ collisions. A 

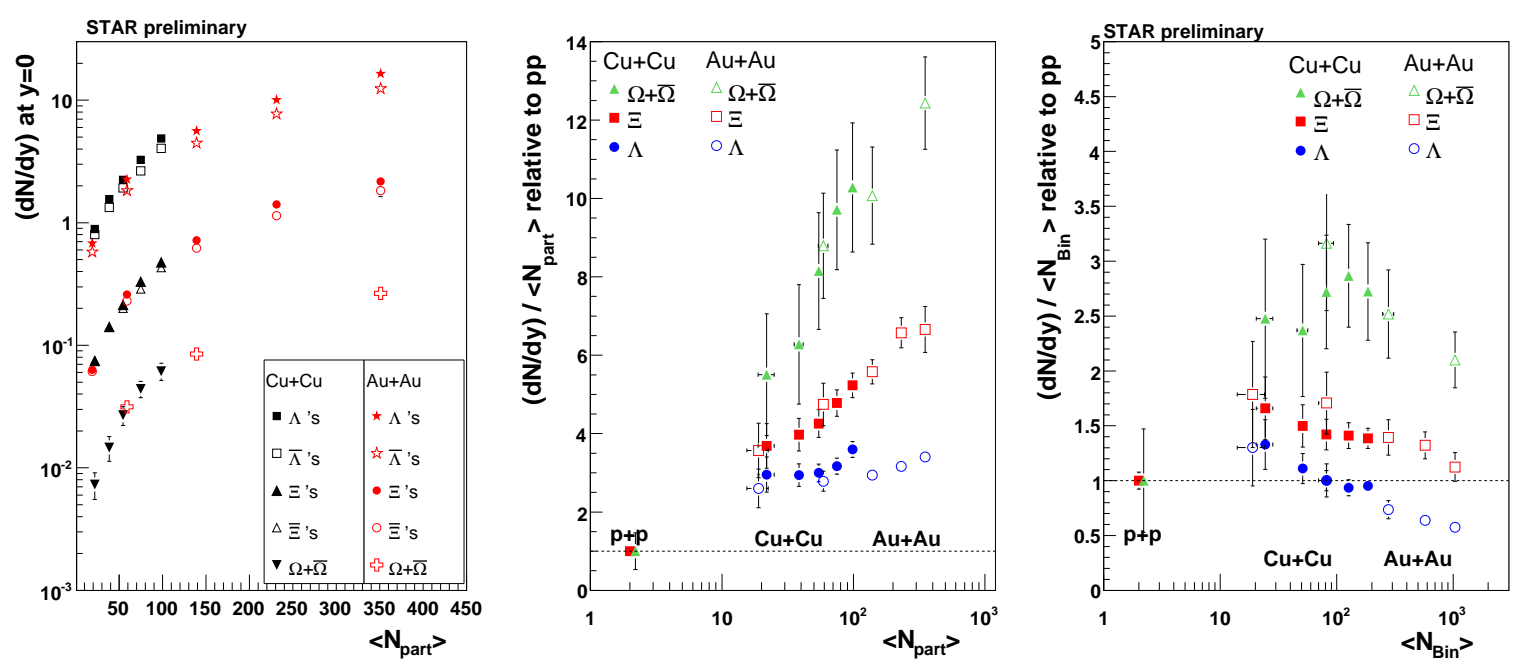

Figure 1. a) Strange particle yields for $\Lambda, \bar{\Lambda}, \Xi^{-+}$and $\Omega^{-+}$as a function of $\left\langle N_{\text {part }}\right\rangle$ for $\mathrm{Au}+\mathrm{Au}$ and $\mathrm{Cu}+\mathrm{Cu}$ collisions. Error bars are statistical only. b) Yields normalized by the collision number of participants $\left\langle N_{\text {part }}\right\rangle$ and c) number of binary collisions $\left\langle N_{b i n}\right\rangle$.

clear enhancement of hyperon production in $\mathrm{Au}+\mathrm{Au}$ and $\mathrm{Cu}+\mathrm{Cu}$ compared to $\mathrm{p}+\mathrm{p}$ can be seen. Anti-Lambda and Anti-Xi yields are not shown in this comparison but similar curves are observed, with slightly lower enhancement levels, which is expected due to non-zero net baryon number. Omega and Anti-Omega yields were added to reduce statistical error. It is interesting to note that this enhancement can be observed even in the most peripheral bins in $\mathrm{Au}+\mathrm{Au}$ and $\mathrm{Cu}+\mathrm{Cu}$ compared to the $\mathrm{p}+\mathrm{p}$ yields. In addition, the enhancement appears to increase linearly as a function of $\left\langle N_{\text {part }}\right\rangle$ and also with strange quark content. The strangeness enhancement hierarchy is in accordance to what is expected from Canonical thermal production [11, but the dependence with volume $\left(\left\langle N_{\text {part }}\right\rangle\right)$ disagrees with this same picture of Canonical thermal production.

Figure 10 shows the strange particle yields normalized by the number of binary collisions $\left\langle N_{\text {bin }}\right\rangle$ and compared to $\mathrm{p}+\mathrm{p}$. Once more, $\mathrm{Cu}+\mathrm{Cu}$ data seems to be in agreement with the $\mathrm{Au}+\mathrm{Au}$ data but with a smaller discrepancy for the $\Lambda$ yield in the most central $\mathrm{Cu}+\mathrm{Cu}$ bin. This plot also shows that strange particle production does not scale with $\left\langle N_{b i n}\right\rangle$, suggesting that a different scaling is required.

A statistical thermal model [12] was used to fit the different particle ratios measured in $\mathrm{Au}+\mathrm{Au}$ and $\mathrm{Cu}+\mathrm{Cu}$ collisions at energies of $200 \mathrm{GeV}$ and $62.4 \mathrm{GeV}$, for different centrality bins. Yields of protons, pions kaons, $\Lambda$ 's and $\Xi$ 's were used to calculate the yields for the fit. Figure 2 shows the results from these fits with chemical freezeout temperature $\left(T_{c h}\right)$, Baryon chemical potential $\left(\mu_{B}\right)$ and strangeness saturation parameter $\left(\gamma_{S}\right)$. The temperature seems to show no dependence on the system size nor on the energy. The baryon chemical potential seems to be constant with sytem size for the $200 \mathrm{GeV} \mathrm{Au+Au} \mathrm{data,} \mathrm{but} \mathrm{shows} \mathrm{a} \mathrm{small} \mathrm{increase} \mathrm{with} \mathrm{centrality} \mathrm{in} \mathrm{the} \mathrm{lower}$ energy $62.4 \mathrm{GeV} \mathrm{Au+Au} \mathrm{data.} \mathrm{The} \mathrm{strangeness} \mathrm{saturation} \mathrm{parameter,} \mathrm{in} \mathrm{the} \mathrm{data} \mathrm{from}$ both energies, shows an increase with the system size till it reaches a saturation point. 

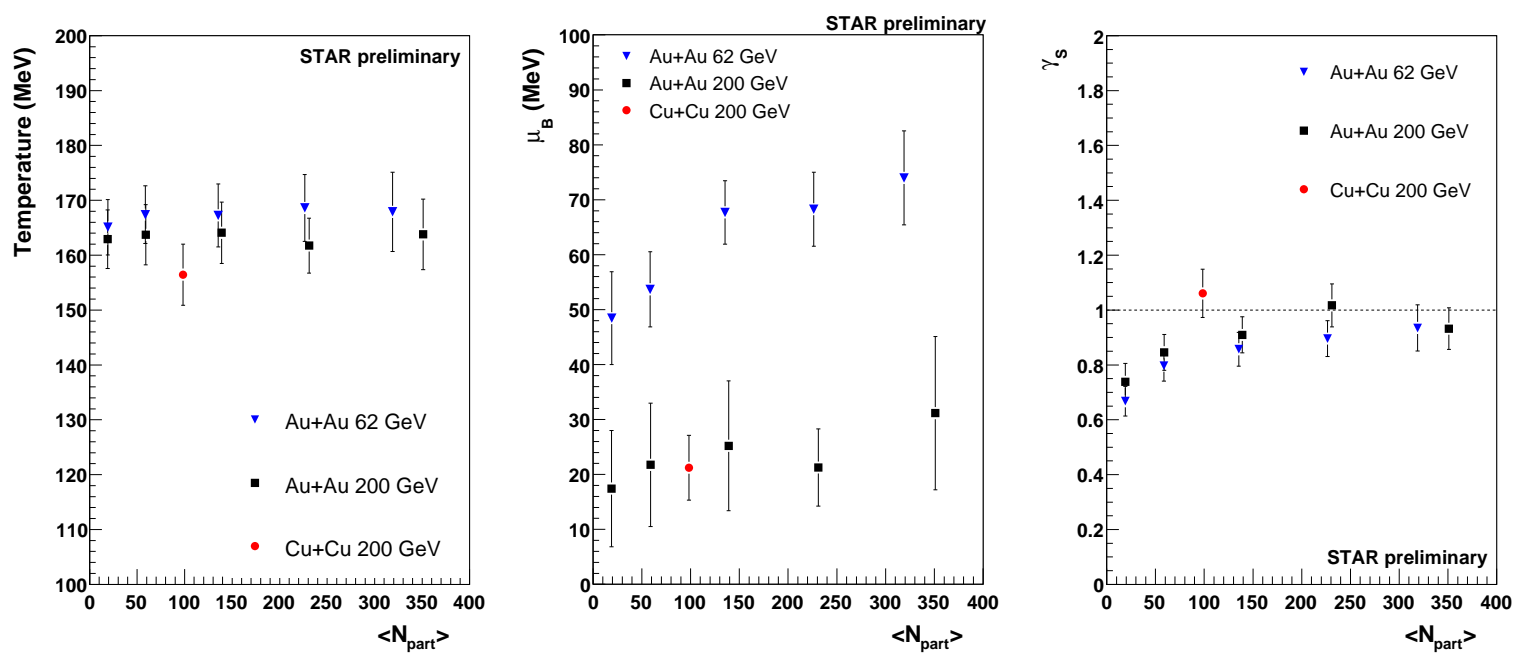

Figure 2. Results from a statistical thermal model fit [12] for $\mathrm{Au}+\mathrm{Au} 62.4 \mathrm{GeV}$ and $200 \mathrm{GeV}$ and $\mathrm{Cu}+\mathrm{Cu} 200 \mathrm{GeV}$ (most central bin only). Chemical freeze-out temperature, baryon chemical potential and strangeness saturation parameter are shown as a function of $\left\langle N_{\text {part }}\right\rangle$.

The new $\mathrm{Cu}+\mathrm{Cu}$ data, was analyzed only for the most central bin, and the comparison in the last plot indicates that strangeness is already saturated for this system, which is not true in the equivalent $\left\langle N_{\text {part }}\right\rangle$ data from $\mathrm{Au}+\mathrm{Au}$ collisions.

\section{Energy Dependence}

Figure $3 \mathrm{a}$ shows the particle yields as a function of collision energy compiling data from experiments at AGS-BNL [13, SPS-CERN [8] and RHIC-BNL [14, 15]. Baryon yields show a strong increase in the region of the AGS collision energies up to around $10 \mathrm{GeV}$, after which, the baryon yields stay relatively constant. Above SPS energies, there seems to be a smooth increase of the baryon production at higher energies. The anti-baryon yields increase continuously and smoothly with energy, that can be inferred from the ratio plots shown in figure $3 \mathrm{~b}$. These plots show the variation of the baryon production mode from a transport dominated regime into a pair-production regime. The new $\mathrm{Cu}+\mathrm{Cu}$ data is included in the ratio plot with a star symbol for $62.4 \mathrm{GeV}$ and $200 \mathrm{GeV}$, and show no difference to the $\mathrm{Au}+\mathrm{Au}$ ratios. From these curves, it is straightforward to predict the expected yields of these particles at the LHC energies. For $\Lambda$ the expected yield is between 10 and 30 , for $\Xi$ it is between 3 and 6 , while for $\Omega$ between 0.4 and 0.7 .

Figure 4 shows an excitation function of the thermal fit parameters $\left(T_{c h}, \mu_{B}\right)$ for the data from the NA49 experiment at the SPS-CERN and STAR-RHIC data. At RHIC energies, the freeze-out temperatures seem to have achieved a saturation point around $170 \mathrm{MeV}$. All fits were done using the THERMUS thermal code [12, using particle ratios calculated from mid-rapidity yields $(\mathrm{dN} / \mathrm{dy})$. Once more, from these plots, it is 

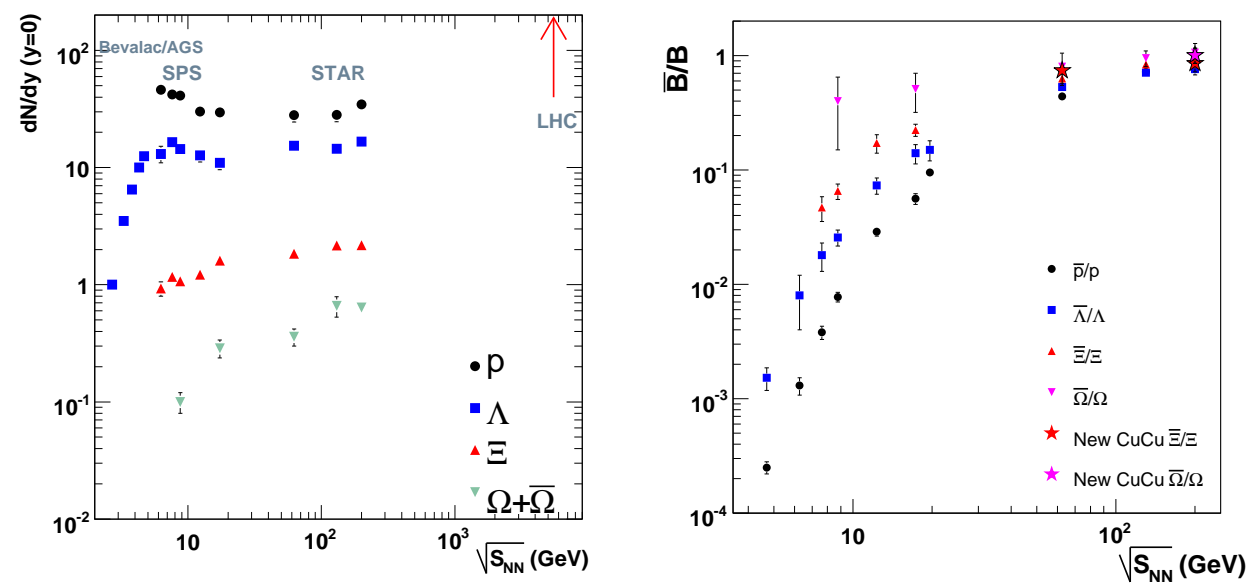

Figure 3. a) Baryon yields as a function of collision energy compiling data from AGS, SPS and RHIC experiments. b) Anti-baryon over Baryon ratio as a function of collision energy. New $\bar{\Xi} / \Xi$ and $\bar{\Omega} / \Omega$ ratios measured in $\mathrm{Cu}+\mathrm{Cu}$ collisions at $62.4 \mathrm{GeV}$ and $200 \mathrm{GeV}$ are included in this systematic and shown as star symbols.
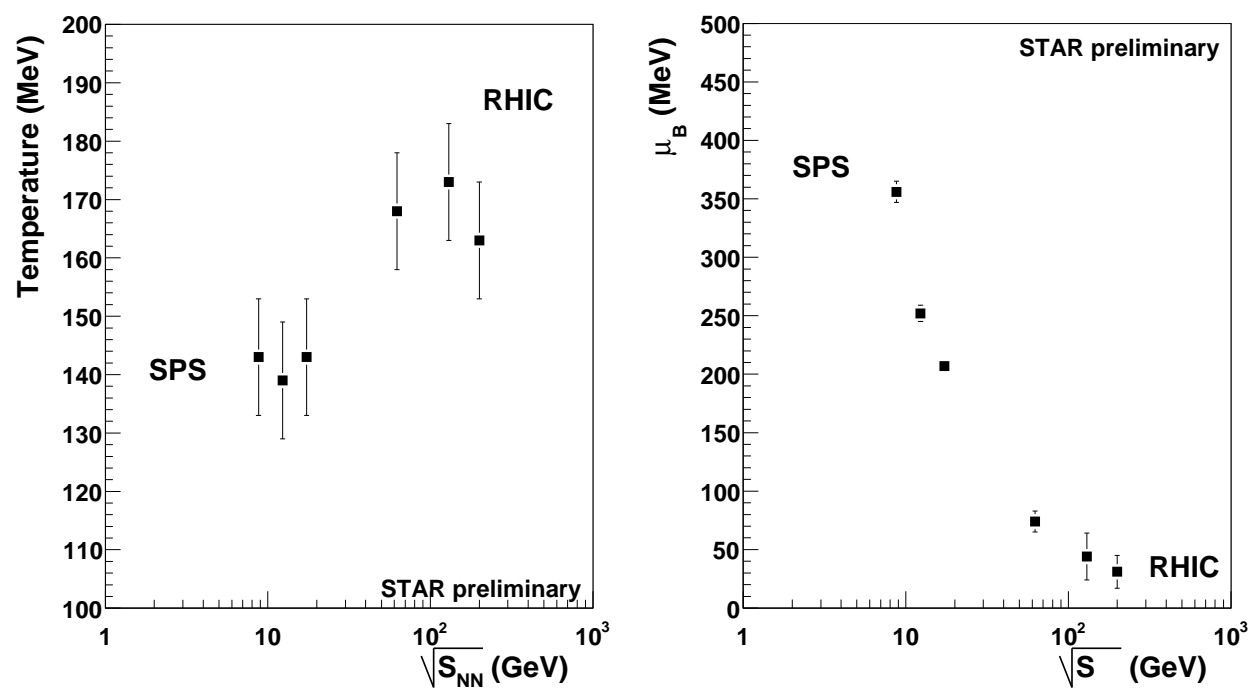

Figure 4. Thermal model fit parameters as a function of collision energy, for SPS and RHIC data points.

possible to predict the conditions expected from the future LHC heavy-ion program, where $\mu_{B}$ should be close to zero and the temperature still around $170 \mathrm{MeV}$.

\section{Conclusions}

There are now considerable amounts of data from heavy-ion reactions that allow for a systematic study of the strange particle production for different energies and different reaction system sizes. This kind of study, and in particular that of strange particle production, is crucial to understand the characteristics of the strongly interacting matter 
formed in RHIC collisions. The comparison between the new $\mathrm{Cu}+\mathrm{Cu}$ data with the $\mathrm{Au}+\mathrm{Au}$ data was shown and seems to be consistent with an $N_{\text {part }}$ scaling, except for the most central bin of the Lambdas. Thus it becomes clear that it is important to find the correct way to compare and scale the various different measurements from different reaction species and energies. The sharp change in behaviour of the particle yields excitation function in the lower energy region has been proposed as a signature for QGP formation [16]. This shows the importance of a high-statistics energy scan as proposed in the next RHIC operation phase. Also, the extrapolation of the various measured curves to the higher energy range shows the importance of the new measurements expected from LHC.

\section{Acknowledments}

We wish to thank Fundação de Amparo a Pesquisa do Estado de São Paulo, and Conselho Nacional de Pesquisa de Desenvolvimento, Brazil for the support to participate in the SQM2007 conference. We thank the RHIC Operations Group and RCF at BNL, and the NERSC Center at LBNL and the resources provided by the Open Science Grid consortium for their support. This work was supported in part by the Offices of NP and HEP within the U.S. DOE Office of Science; the U.S. NSF; the BMBF of Germany; CNRS/IN2P3, RA, RPL, and EMN of France; EPSRC of the United Kingdom; the Russian Ministry of Sci. and Tech.; the Ministry of Education and the NNSFC of China; IRP and GA of the Czech Republic, FOM of the Netherlands, DAE, DST, and CSIR of

the Government of India; Swiss NSF; the Polish State Committee for Scientific Research; Slovak Research and Development Agency, and the Korea Sci. \& Eng. Foundation.

\section{References}

[1] Karsch F. 2002 Nucl. Phys. A 698199

[2] Rafelski J. 1991 Phys. Lett. B 262333

[3] Rafelski J. and Mueller B. 1986 Phys. Rev. Lett. 562334

[4] Harris JW. et al. (STAR Collaboration) 2002 Nucl. Phys. A 698 64c-77c

[5] Admas J. et al. (STAR Collaboration) 2004 Phys. Rev. Lett. 92182301

[6] Adler C. et al. (STAR Collaboration) 2002 Phys. Rev. Lett. 89092301

[7] Takahashi J. 2005 Journal of Phys. G31 S1061

[8] Anticic T. et al. (NA49 Collaboration) 2004 Phys. Rev. Lett. 93022302

[9] Antinori F. et al. (NA57 Collaboration) 2006 Jornal of Phys. G 32 427-442

[10] Timmins A. 2007 These proceedings.

[11] Tounsi A. and Redlich K. 2004 arXiv:hep-ph/0111159

[12] Wheaton S. and Cleymans J. 2004 arXiv:hep-ph/0407174

[13] Albergo S. et al. (E896 Collaboration), 2002 Phys. Rev. Lett. 88062301

[14] Adams J. et al. (STAR Collaboration) 2003 Phys. Lett. B 567 167-174

[15] Adler C. et al. (STAR Collaboration) 2001 Phys. Rev. Lett. 864778

[16] Gazdzicki M. and Gorenstein M.I. 1999 Acta Phys. Polon. B30 2705 